\title{
Optimizing developmental outcomes by setting smart goals individualized home program for children with disabilities during COVID-19
}

Aarti Nair *1, Diya Nair ${ }^{2}$, Megha Girdhar ${ }^{3}$, Anchit Gugnani ${ }^{4}$.

${ }^{{ }^{*}}$ PhD Scholar, NIMS College of Physiotherapy and Occupational therapy, Jaipur, Rajasthan, India.

${ }^{2}$ Speech \& Language Pathologist \& Audiologist, Latika Roy Foundation, 113/1 Vasant Vihar. Dehradun, 248006. India.

${ }^{3}$ Monitoring \& Evaluation Head, Latika Roy Foundation, 113/1 Vasant Vihar. Dehradun, 248006. India.

${ }^{4}$ Associate Professor, NIMS College of Physiotherapy and Occupational Therapy, Jaipur, Rajasthan, India.

\section{ABSTRACT}

Background: The recent flare-up of the COVID-19 pandemic has posed a huge danger to the lives and prosperity of millions of individuals around the world. The weakest segments of society are being missed in this largescale crisis. As the world opens up from lockdowns and enters an unprecedented condition of uncertainty, or what many have called "the new normal", it bodes well to ponder what are the lessons learnt. Understanding such risks and assessing the socioeconomic impact of the pandemic are key to shaping a response that considers the needs of all children. The Covid pandemic presents a phenomenal chance for the families of children with disabilities to act in fortitude and transform this emergency into a force to accomplish the objectives and enhance result for their children.

Methods: Children and young adults in the age group 1-21 years were part of this program. A total of 261 participants have undertaken the services provided by LRF staff during the year long period from Jan -Dec 2020.

Results: In this article, we feature the home management programs that were setup by the staff at Latika Roy Foundation (LRF) during the pandemic for families of children with disabilities. Families of children with disabilities worked hard during the lockdown and their children showed improved outcomes by the individualised home programs that were set for each child attending the online services

Conclusion: The pandemic led to shift from regular services to online mode of service and displayed clearly that necessity is the mother of invention. When accessibility to essential services was difficult, children with disabilities and their parents were the high-risk group for various physical and mental health issues and needed appropriate guidance and support. As the world navigates the pandemic the LRF staff continued to work for the most vulnerable sections of society fostering the idea of practice and participation.

KEY WORDS: Pandemic, the new normal, families of children with disabilities, online services.

Address for correspondence: Dr. Aarti Nair, PT Latika Roy Foundation, 113/1 Vasant Vihar. Dehradun, 248006. India. E-Mail: aarti@latikaroy.org

Access this Article online

Quick Response code

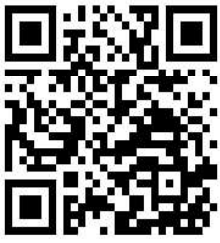

DOI: $10.16965 /$ ijpr.2021.184
Journal Information

International Journal of Physiotherapy and Research

ISSN (E) 2321-1822 | ISSN (P) 2321-8975

https://www.ijmhr.org/ijpr.html

DOI-Prefix: https://dx.doi.org/10.16965/ijpr

Article Information
Received: 16 Aug 2021

Peer Review: 16 Aug 2021

Revised: None
Accepted: 21 Sep 2021

Published (O): 11 Oct 2021

Published (P): 11 Oct 2021 


\section{BACKGROUND}

Since schools closed and lockdown began, states and providers have been grappling with how to support children with disabilities in a virtual environment. Parents and caregivers of children with disabilities are generally experiencing higher rates of stress, depression and anxiety than other parents [1]. The weakest segments of society are being missed in this largescale crisis [2]. As the world opens up from lockdowns and enters an unprecedented condition of uncertainty, or what many have called "the new normal", it bodes well to ponder what we have learnt. Understanding such risks and assessing the socioeconomic impact of the pandemic are key to shaping a response that considers the needs of all children [3]. The Covid pandemic presents a phenomenal chance for the families of children with disabilities to act in fortitude and transform this emergency into a force to accomplish the objectives and enhance result for their children [4].

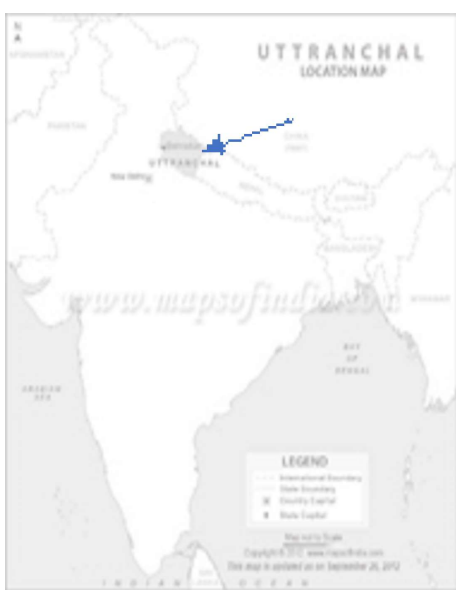

As Covid-19 pandemic continues staff at Latika Roy Foundation, Dehradun continues to provide services for learning, therapy and wellbeing during school closure. LRF is an NGO established in 1996, providing services for the weakest sections of society in the Dehradun district of Uttarakhand.

How to manage during this unexpectedly long and challenging stay at home is a question all of us are struggling to answer. This ongoing crisis is far more serious and has got further complicated leading to parents enduring high stress [5]. Supporting children with disabilities at home during the lockdown requires extraordinary effort and patience [6].
Under normal circumstances, parents' burden would be shared by centres, inclusive school and vocational centers. Living in confined space without social interaction for long periods of time has intensified existing challenging behaviours [7]. Presently, managing these behaviours at home is falling solely on parents. Interruption of requisite therapies can have long-term consequences [8] on children with development disabilities. Discontinuing regular therapy may worsen functional ability and cause complications in children with developmental delays.

Aims and Objectives: With the vision of providing support to children and families with disabilities, staff at LRF developed individual home programs for children, set up specific goals and continued online services to optimise development of children during this pandemic. Perception of delay in child's progress, inaccessibility to remedial services along with economic constraints due to lockdown and inability to engage children in meaningful activities throughout the day imposes a huge mental burden. The staff addressed the need to maintain the mental health of parents/ caregivers of children with special needs while taking care of their children.

To achieve the requisite goals, require a full-time team working closely with families at home. Bulk of the work was done at home by parents and other family members. Training parents to work effectively with their children, keeping them motivated and convincing them to commit to doing regular therapy on their own was a constant effort.

- Optimizing development outcomes by setting SMART goals.

- Providing individualized home based programs to empower parents and reduce stress.

\section{METHODOLOGY}

Population and Sample: Children and young adults in the age group 1-21 years were part of this program. A total of 261 participants have undertaken the services provided by LRF staff during the year long period from Jan -Dec 2020. The participants included family members, usually the mother, sometimes fathers, sibling and 
grandparents of the children with disabilities enrolled for sessions in LRF.

Data and Sources of Data: LRF uses Salesforce as its Management Information System (MIS) and central data repository. It is used efficiently to track the progress of the children / young adults during their time with us. The data for this paper has been retrieved from Salesforce.

Theoretical framework: The Covid-19 pandemic affected staff and stakeholders at LRF as it did the rest of the world. This unprecedented situation required the organization to take prompt, clear organizational decisions. In view of the vulnerable children, we work with, LRF was the first NGO in Dehradun to take the forward-looking decision of closing its centres from $15^{\text {th }}$ March 2020 for children and staff. The teams and staff were shifted to working from home for providing online services for children.

As the first measure towards preparing the staff to work from home and follow all necessary guidelines the Covid-19 Risk Index was shared with all the staff and parents. It was translated in Hindi to make it clearer and reader friendly.

Children with special needs were and are facing additional challenges of understanding and dealing with the difficulties brought about by the ongoing pandemic due to their uncertain conditions. To mitigate these challenges staff started with providing services through Videos. In the beginning we designed simple videos on cleanliness for children and regular washing of hands.

The fact that children with disabilities tend to have a weak immune system raises the risk by many folds and makes them more susceptible to infection ${ }^{1}$. Therefore, complete care needed to be taken up to keep their bodies clean. The tendency of children to touch surfaces and handle things without much concern for hygiene added to increased risk. It was therefore extremely important to sanitize their hands properly and frequently.

Most children with disabilities have difficulty in following instructions and learning from experience. As most children were good at imitation, appropriate activities were shown through visuals. Caregivers were given videos so that instructions could be repeated.
Orchestrating the services: Besides other responsibilities at home, managing and coordinating various activities was one of the main challenges faced by young parents [2]. Most homes have one smart phone and very few have computers. Juggling online services while parents had to manage their work, including the typical child's online school work and the special child's therapy sessions a task by itself. Internet connectivity issues, getting used to technology platforms added to the existing challenges the team and parents faced during the first quarter of April-June 2020. By repeated team meetings, practice sessions on Google and Zoom meets and technology support to each-other, the staff and parents circumvented these challenges.

Adjusting to new normal: As daily therapy got confined to homes, therapy had to be modulated and adapted to avoid decline in child's development. Activities of daily living, brushing, combing, wearing shoes amongst many others were all structured to become exercises. Small responsibilities were assigned to children as part of their daily routine not only to increase participation but also to build the concept of responsibility. The only difference in these activities was that they were assisted by parents in a manner that they became active assistive exercises which in turn was therapy for that day.

Virtual parent-teacher meetings (PTMs) were conducted for all children/young adults in May 2020. Our teams embraced technology, learnt by doing, as did our parents and a lot of work has been covered over the course of the last months.

From the month of August 2020, as children and families got more comfortable with using technology platforms, structured individual and group virtual classes were started. A time-table similar to when the school was open was followed. A few families who do not have smart phones, continue to receive follow-up sessions over phone calls.

Entertainment and Therapy: Listening to music, watching a movie or dancing all became exercises. While children listened to music or watched a movie, posture control and alignment 
was maintained, allowing both exercise and entertainment simultaneously. Sugar-coating exercises with fun and play made it easy for parents to encourage their children to engage in otherwise tedious activities. This has become the "new normal".

Dealing with challenging behaviours: Absence of a daily timetable can be trying for youngsters with intellectual disabilities. They require schedules and structure and least disruptions in their routine. Absence of comprehension of the impacts of pandemic, protection from change and failure to adjust to new methodologies prompted previous conduct issues heightening or advancement of novel ones in these youngsters, particularly those with mental handicap. Kids with attention deficient and hyperactivity disorders (ADHD) and learning issue were unable to utilize online school meetings because of decreased capacity to focus or trouble in cognition.

Dealing with challenging behaviours especially during Covid-19 pandemic has been extremely tough. A daily work-flow structure was outlined by each case-manager. Every child/young adult was called on the phone once a week by the respective Special Educator and Therapist. In the initial sessions, the importance of maintaining a daily schedule for each child was stressed along with repeated learning on hand-washing and wearing face-masks. Many videos and messages on hand washing, mask-wearing for children were created by the teams.

Even the best-intentioned families had difficulty in meeting behaviour challenges of their children because of limited skills and resources [9]. Knowing that every behaviour is a form of communication, and most families were unclear on how to deal with this form of communication. $V$ isual time-tables were created by Special educators for parents and children to help them follow a structured, at-home, learning routine. The Physiotherapists worked on engaging the parents to work on functional therapy for their children using home-based resources. Key workshop sessions included creating social stories, wearing masks correctly

Maintaining emotional wellbeing: As lockdown continues and gets prolonged, motiva- -tion is decreasing, and parents are getting exhausted and depression is setting in. Their children's development related stress which was earlier shared between parents, schools and therapy centres, has to be dealt with by them alone. Thus, emotional well-being of caregivers is imperative. Resolving these uncertainties and providing needed supports to reduce parents' stress is a priority.

The team of counsellors worked hard as families dealt with the additional stress of the forced quarantine. Individual counselling sessions to mothers, fathers, and trainees were planned.

Support group were setup through WhatsApp and zoom meetings were done. Sessions on positive parenting approach, behaviour management, inclusion, financial security and social stories were facilitated.

Parents are encouraged to maintain some schedule for their children by following online school sessions and engaging them in fun-based household chores. Wherever possible, children are encouraged to continue social interaction through supervised telephonic and video calls. Avoiding extra demands and unrealistic expectation from children during these times has helped in eluding frustration and behavioural issues.

Statistical tools and econometric models: Assessment is the process of gathering information about the strengths of the child and the areas where a child faces challenges or needs help. Children with disabilities often have a disjointed development, which similarly affects their functional adaptive ability, e.g. a child may be good in math skills but delayed in social skills. Assessment on a standardised functional skills checklist ensures that students are assessed for all domains or areas of development.

SMART is a well-established tool that you can use to plan and achieve your goals. While there are a number of interpretations of the acronym's meaning, the most common one is that goals should be Specific, Measurable, Achievable, Relevant, and Time-bound.

SMART goals help to create clear, attainable and meaningful goals, and develop the motivation, action plan, and support needed to achieve 
them. A goal is an idea of the future or desired result that a person or a group of people envision, plan and commit to achieve. People Endeavour to reach goals within a finite time by setting deadlines. SMART is a well-established tool that you can use to plan and achieve your goals. While there are a number of interpretations of the acronym's meaning, the most common one is that goals should be Specific, Measurable, Achievable, Relevant, and Timebound.

Descriptive Statistics: 261 participants who received regular online sessions during the pandemic were accounted for. A qualitative analysis was conducted for the following study. $27.2 \%$ or participants accounted for those with cerebral palsy, $13.7 \%$ with Down's syndrome and $13.6 \%$ with autism making these the most prevalent disorders seen amongst participants (Ref:Figure1). The data was arranged based on various properties and characteristics seen at the organization. The purpose of following qualitative statistics for the current study was to capture various changing attributes over the selected time period. Since the data is based on markers mentioned above, it provided a more flexible approach to consider optimization of SMART Goal setting.

Ethical Approval and Consent: Before this study began, ethical approval was obtained from LRF's ethical committee (vide letter No. (01E/LRF) dated 27/06/2021). Prior to the commencement of the study, each participant was explained the purpose, aims and objectives of the study and thereafter their written consent was obtained and documented.

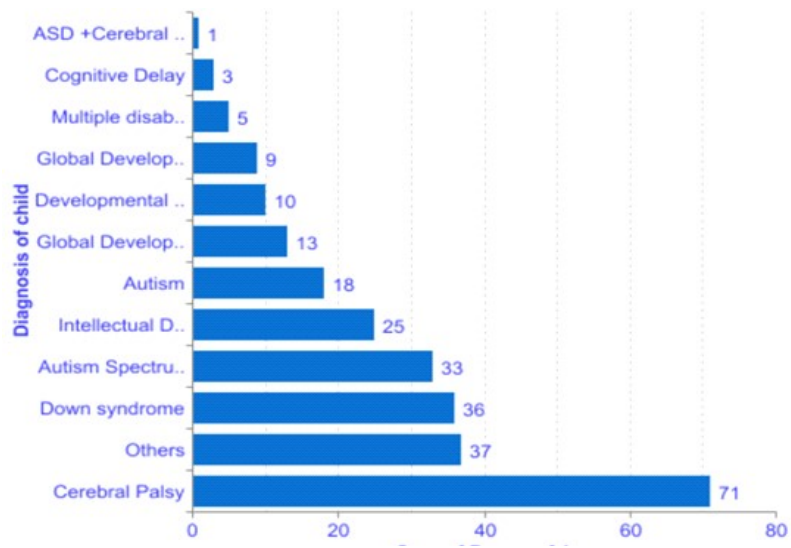

Figure 1: The above graph depicts the different range of disabilities of the 261 participants who received regular online services during the period of Jan-Dec 2020.

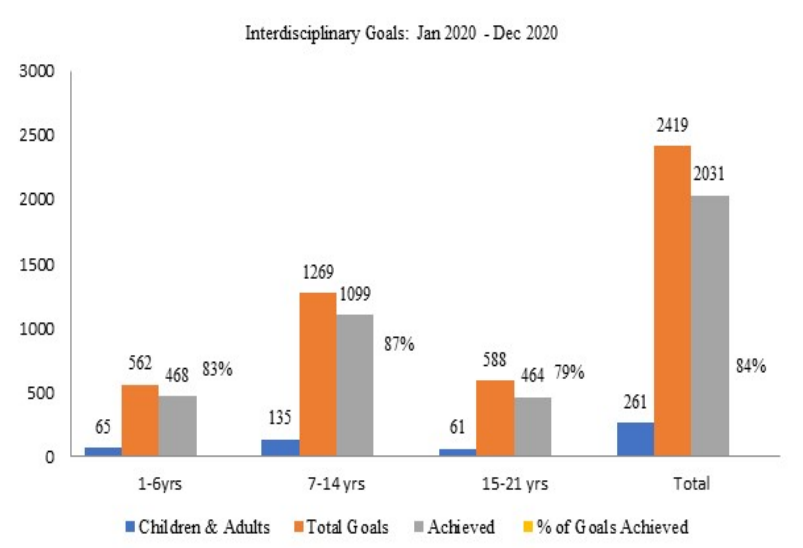

Figure 2: The following graph further summarizes the work and output numbers of online services provided across Quarters 1,2 and 3, till Dec 2020.

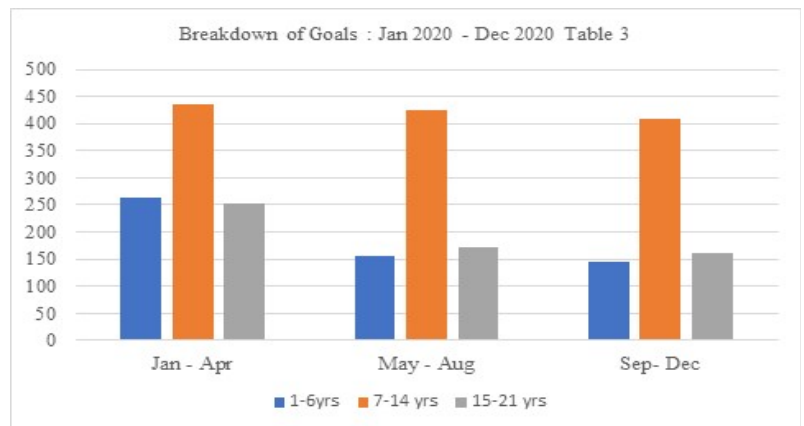

Figure 3: shows the breakdown of interdisciplinary goals set for the 261 participants through the year Jan-Dec 2020. A total of 562 goals were set for children in the age group of 1-6 years, 262 in the first quarter, 156 in the second quarter and 144 in the third quarter. A total of 1269 goals were set for children in the age group of 714 years, 425 in the first quarter, 426 in the second quarter and 408 in the third quarter. For the age group of 1521 years, a total of 582 goals were set for young adults, 253 in the first quarter, 173 in the second quarter and 162 in the third quarter.

Table 1: Percentage of goals achieved - Jan 2020 - Dec 2020.

\begin{tabular}{|cccc|}
\hline Age & Jan -Apr 2020 & May-Aug 2020 & Sep -Dec 2020 \\
\hline 1-6 years & $78 \%$ & $88 \%$ & $88 \%$ \\
\hline 7-14 years & $85 \%$ & $85 \%$ & $88 \%$ \\
\hline 15-21years & $69 \%$ & $86 \%$ & $88 \%$ \\
\hline
\end{tabular}

The percentage of goals achieved during the months before online services began was calculated in the months Jan-Apr 2020. The percentage of goals achieved during the months May-Aug2020 and Sep-Dec 2020 was when services were provided through virtual sessions. An effort was made to support parents and help them achieve the goals keeping in mind the existing challenges an stress every family was undergoing. 


\section{RESULTS AND DISCUSSION}

With the emerging situation of working from home for staff and the challenges above, monitoring the work progress and data recording had to be adapted to an easily accessible and userfriendly interface. Google data-matrix sheets were developed to record the progress with each child/young adult over the phone-sessions across Quarter 1, Jan-April 2020, Quarter 2, MayAug, and Quarter3, Sep-Dec 2020 (Figure 2). The data-matrix was linked with the unique child IDs that were subsequently useful to upload and integrate onto the LRF database in Salesforce. This enabled to maintain the continuity of dataflow despite all teams working from home during the lockdown.

In our country where the pandemic is creating havoc and the infrastructure is doing its best to cope, LRF brings hope that we can continue to make lives better for families of young children with disabilities, by helping parents and caregivers with some basic intervention strategies that they can do, within the chaos that our lives have become. The online services, are a space to feel surrounded by peers, to feel supported, understood and to realize their incredible potential. So, rally on as our mission is to make a better place for people with disabilities.

Constant re-evaluation of our approach and relying on feedback from the families has helped us improve. Parents have always been incredibly innovative and resourceful in adapting their home environments and using ordinary household items for therapeutic activities. Covid 19 has been a kind of testing ground for the faith we have always had in them as agents of change. In some ways this lockdown has been a boon in disguise. All parents are working in creative ways with their kids. Parents are involved. Fathers as well. The therapists' group were amazed to see ways in which parents were managing their kids at home. Challenging but remarkable.

$27.2 \%$ had cerebral palsy, $13.7 \%$ has down syndrome, $12.6 \%$ had ASD, $6.8 \%$ had autism and 9.5\% had intellectual disability. These children received special education services, speech and language and physiotherapy services which were designed based on their functional levels that were determined by FACP. Infants and toddlers received early intervention services at home.

Among children aged 1-6years more than $80 \%$ achieved the goals that were formulated for them. In the age group of $7-14$ years, $87 \%$ of the children achieved the Interdisciplinary goals set for them while the age group, 15-21years, 79\% achieved the goals. (Figure:3, Table 1)

\section{CONCLUSION}

Considering the challenges faced by the entire world during Covid19 pandemic especially for those with disability, creating SMART goals was considered one of the better approaches to streamline online intervention for children and support families. The bifurcation of these goals into its constituent divisions enabled professionals to measure and in turn modify any changes in the goals as communicated by parents who were considered as the key for supporting their children. This approach led to a decrease in parental stress, further promoting efficacy of online therapy using SMART goals. The individualized programs helped parents to understand the steps towards development and lead to reduced stress. Home-based programs added valuable insights to their creativity and parents felt supported and empowered.

The parents have become key facilitators in this approach and the teams see parents gaining more and more confidence in working with their children at home each week. To summarize, during the current pandemic when accessibility to essential services is difficult, children with disabilities and their parents are a high-risk group for various physical and mental health issues and need appropriate guidance and support. Emotional wellbeing is therefore the essence of meaningful intervention services leading to improved quality of life during the pandemic.

\section{ABBREVIATIONS}

LRF- Latika Roy Foundation

CRM- Customer Relationship Management

MIS- Management Information Systems

ADHD- Attention Deficient Hyperactivity Disorder

PTM- Parent Teacher Meeting

NGO- Non-governmental organization 
SMART- Specific, measurable, achievable, repetitive, time bound

FACP- Functional Assessment Checklist for Programming NIEPID-National Institute for Empowerment of Persons with Intellectual Disabilities

\section{ACKNOWLEDGEMENTS}

The field work of this study was made possible through staff of Latika Roy Foundation. I would like to thank all the 36 staff members who were actively involved in the functioning and gathering the data for evaluating the program. Especially because they continued to work during the ever changing and extremely challenging time of Covid 19Pandemic. Secondly, I wish to thank the families who agreed to be part of this study from the District of Dehradun. I would like to express my deep and sincere gratitude to all of them. I will always be grateful to the Executive Director of Latika Roy Foundation, Jo Chopra, who has had faith and confidence in my ability and was extremely supportive throughout the difficult times, especially with restrictions imposed due to the Covid Pandemic. I would like to thank her for her friendship and empathy. I would like to thank all my friends and colleagues who have supported me and provided help when I needed it the most. I wish to mention the Chief Operating Officer of Latika Roy Foundation, Ms. Sumita Nanda, Mr. Sandeep Khanna, Project Head, Gubbara, Mr. Rizwanali, Senior Advocate, who were ever ready to help. Thank you to my family and friends. Thank you to my parents who have encouraged me and provided all the support while I completed my work.

\section{Conflicts of interest: None}

\section{REFERENCES}

[1]. The latest COVID-19 news and resources, as well as tips to protect you and your family. UNICEF

[2]. Leaving no one behind during the pandemic: Children with disabilities and COVID-19, Pg 4

[3]. Leaving no one behind during the pandemic: Children with disabilities and COVID-19 Back to Unicef.org.
[4]. Craft, A. Childhood in a digital age: Creative challenges for educational futures. London Review of Education, 2021;10(2):173-190.https://doi.org/ 10.1080/14748460. 2012.691282.

[5]. Patel, Khushboo, 'Mental Health Implications of COVID-19 on Children with Disabilities', Asian Journal of Psychiatry, 2020;54.

[6]. Aishworiya, Ramkumar, and Ying Qi Kang, 'Including Children with Developmental Disabilities in the Equation during this COVID-19 Pandemic', Journal of Autism and Developmental Disorders 2020.

[7]. WHO Disability considerations during the COVID19 outbreak. Available from: https://www.who.int/ publications/i/item/disabilityconsiderations-during-the-covid-19 outbreak. Accessed June 5, 2020

[8]. Nancy A. Murphy, Paul S. Carbone and the Council on Children With Disabilities Pediatrics October 2011;128(4):795-802. DOI: https://doi.org/10.1542/ peds.2011-1467

[9]. https://content.iospress.com/articles/journal-of-pediatric-rehabilitation-medicine/prm200769\#

[10]. Children with disabilities in the United States and the COVID-19 pandemic.

[11]. Chuanmei Dong, Simin Cao, Hui Li. Children and Youth Services Review 2020;118.

How to cite this article: Aarti Nair, Diya Nair, Megha Girdhar, Anchit
Gugnani. Optimizing developmental outcomes by setting smart goals
individualized home program for children with disabilities during COVID-
19. Int J Physiother Res 2021;9(5):4028-4034. DOI: $10.16965 /$ ijpr.2021.184 